\title{
Quality control of hardening technologies using the acoustic emission method
}

\author{
E.V. Grigoriev, A.G. Palaev, T.S. Golikov, V.V. Nosov
}

St. Petersburg mining University, Saint Petersburg, Russia

\begin{abstract}
The ways of increasing the reliability and service life of industrial facilities are considered. The types of hardening technologies and methods of their control are analyzed. An approach that makes it possible to evaluate the quality of hardening technologies based on the registration of acoustic emission (AE) is described. The results of experimental research on the quality of three types of strengthening technologies are presented and the effectiveness of the proposed approach is shown. The obtained results can be used to assess hardening technologies at real facilities in a non-destructive way.
\end{abstract}

\section{1 introduction}

Due to the increasing requirements for the reliability of technical devices, the need to improve technologies aimed at bettering their operational properties is becoming more and more urgent. In particular, there is a large number of various hardening technologies applied in practice [1], which can affect the entire volume of a product or its individual parts. Each of the existing hardening methods has its own advantages and disadvantages, which are characterized by specific technical and economic indicators that determine the effective areas of application of hardening technologies. Both traditional, well-known technologies are being improved, and fundamentally new technologies are being developed (ultrasonic, laser, electrophysical, etc.), which makes it possible to affect the entire volume of the product or to obtain thin coatings [2,3] (or layers) on a variety of materials. The effectiveness of applying one or another hardening technology on a particular product depends on many factors and has to be ensured by methods of quality control, which is an important task [4].

Quality control of hardening technologies used at real facilities should be carried out promptly and without destroying the product material, which ultimately boils down to a non-destructive assessment of the strength or degree of hardening of the processed elements [5,6]. Particularly, hardening technologies can be used to reduce residual stresses in the material [1], which always contributes to an increase in the resource of the control object. Therefore, the issue of controlling 
the degree of hardening of a particular hardening technology by a fast and non-destructive method remains relevant.

The existing methods for estimating the quality of hardening technologies can be classified in terms of the type of the monitored signal and its connection with the processes that determine the strength of the material. The signals recorded by methods of transmission and reflection of waves introduced from the outside are indeterminately related to the strength and the process of damage growth, since nano-scale factors are not taken into account due to the bending of wave round strength anomalies. Unfortunately, traditional physical methods of non-destructive testing (for instance, ultrasonic) do not allow to detect defects less than a quarter of the ultrasonic wavelength in the controlled material, i.e., less than 2-5 mm [7]. The methods of irradiation are quite promising, since they emit waves associated with the process of material destruction. These are electromagnetic and acoustic emissions. Examples of assessing the quality of hardening technologies using the acoustic emission method are given in [8-11]. The paper proposes a different perspective on the use of this method.

The purpose of this work is to substantiate an operational and non-destructive method of quality control of hardening technologies based on an information-kinetic approach to the interpretation of the results of signal registration, performed using the example of welded joints.

\section{Acoustic emission method and information-kinetic approach to interpretation of results of signal registration}

The method of acoustic emission (AE) is a passive method of acoustic non-destructive testing, based on the registration of acoustic waves emitted by the object of control (OC) under the influence of external stress.

The main problem of the strength AE control is associated with the complexity of the interpretation and quantitative correlation of the results of recording AE signals with the strength characteristics. AE signals are noise-like; extracting a useful signal from noise is a complex procedure. The scheme of the informative AE control is shown in Figure 1.

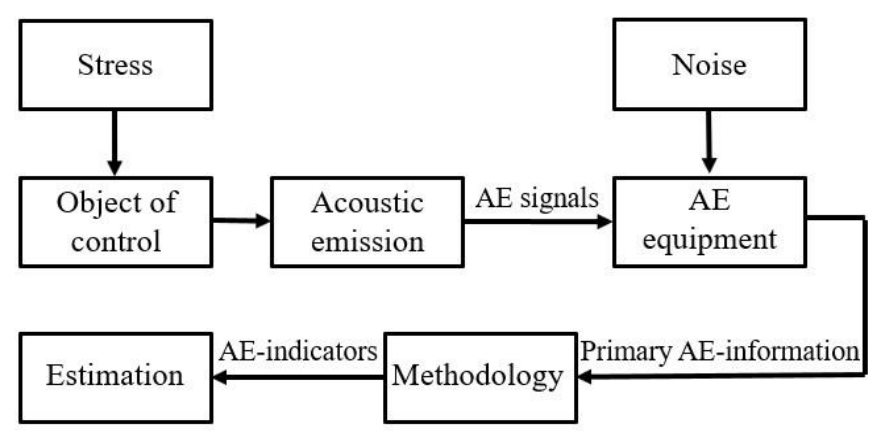

Fig. 1. Scheme of acoustic emission monitoring.

One of the solutions to the problem of controlling the strength AE is the use of an informationkinetic approach based on a multi-level model of acoustic emission parameters, combining statistical and physical approaches to diagnosis, as well as additional "scooping" information on the macro-, micro-, and nano-levels of strength studies to justify the choice of valuable diagnostic indicators of AE. A more detailed approach is described in [12].

Each object consists of structural elements, the state of which determines the state of the whole object. It is known [12] that most of the elements do not carry overloads, while a smaller part of 
them is overloaded to one degree or another. At a certain point in time $\mathrm{t}=\tau^{*}$, the concentration of overloaded structural elements $\mathrm{C}$ reaches the critical value $\mathrm{C}^{*}$, at which the destruction of each structural element has already significantly affected the stress state around the neighboring, previously destroyed. The process of destruction of structural materials is being presented in two stages. At the first stage, two-phase dispersed destruction occurs: inhomogeneous and homogeneous. At the stage of inhomogeneous destruction, structural elements are destroyed throughout the entire volume of the object at different rates, which manifests itself in an increase in the concentration of microcracks in the material. In this case, the heterogeneity is of stochastic nature and is associated with a spread in the mechanical properties of structural elements - grains, random packing of fibers, initial breaks and bends, etc. At a certain moment, the structural elements combine into clusters (areas of broken bonds), the sizes of which are related to the sizes of the combined structural elements. If the cluster sizes are not dangerous, a further larger-scale stage of dispersed destruction occurs, and these stages replace each other until the size becomes dangerous. Further destruction becomes localized, reaching the $\mathrm{C} *$ value, and occurs in the form of nucleation and crack growth.

The decisive moment of destruction is the rate of accumulation of the concentration of destroyed elements. Using the acoustic emission method, a search for structural elements that collapse at the same rate (homogeneous stage) is made. It is this stage of destruction that determines its further behavior, which makes it possible to predict the residual resource.

Considering the heterogeneity of the strength state of the material, the equation of the rate of growth of the concentration of the destroyed structural elements has the form:

$$
C(t)=C_{0} \int_{\omega_{0}}^{\omega_{0}+\Delta \omega} \Psi(\omega)\left\{1-\exp \left[-\int_{0}^{t} \frac{d t^{\prime}}{\theta}\left(U_{0}, \omega\left(t^{\prime}\right)\right)\right]\right\} d \omega,
$$

where $C_{0}$ is the initial concentration of structural elements in the material before failure; $\omega_{0}$ is the minimum value of the parameter $\omega$ of the function $\Psi(\omega)$ of the distribution of its values among structural elements; $\Delta \omega$ is the confidence interval of the scatter of $\omega$ values over structural elements; $\Psi(\omega)$ is the density function of the distribution of the values of $\omega$ over structural elements; $\theta\left(U_{0}, \omega\left(t^{\prime}\right)\right)=\tau_{0} \exp \left\{\left[U_{0}-\gamma \sigma\left(t^{\prime}\right)\right] /(K T)\right\}$ is the formula of S. N. Zhurkov; $\mathrm{U}_{0}$ is the activation energy of the destruction process; $\omega=\gamma \sigma / \mathrm{KT}$ is the parameter of the strength state of the structural element; $\gamma$ is the activation volume (nanostructure parameter); $\sigma$ is the tensile stresses on the structural element; $\mathrm{K}$ is the Boltzmann constant; $\mathrm{T}$ is the absolute temperature; $\tau_{0}$ is the period of atomic vibrations $\left(=10^{-12} \ldots 10^{-14}\right)$.

The multilevel model of time dependences of the AE parameters has the form:

$$
\xi(t)=k_{A E} C(t)
$$

where $\xi$ is the informative AE parameter (number of pulses, amplitude, total amplitude, energy); $\mathrm{k}_{\mathrm{AE}}$ is the acoustic emission coefficient (AEC).

The meaning of the coefficient is that it is related to the fraction of the AE signals from the total pulse stream that has passed time, frequency, and amplitude filtering. The AEC has the form:

$$
k_{A E}=V \iiint_{\Delta t, f, u} \Phi(\Delta t, f, u) d u d f d \Delta t
$$

where $\mathrm{V}$ is the controlled volume of material; $\Phi(\Delta t, f, u)$ is the probability density of the distribution of the AE signals over the intervals $\Delta t$ between them, the frequency $f$, and the amplitude $u$. Taking into account the stochastic nature of elastic radiation, the triple integral in the expression can be used to give the sense of the probability of recording, i.e., the probability that the parameters of elastic waves arriving from the $\mathrm{AE}$ source fall into the range of frequencies, amplitudes of the AE signals, and time intervals between them recorded by the measuring equipment.

By linking the parameters of acoustic emission $(\xi)$ with the parameters of microscopic destruction $(\mathrm{C}(\mathrm{t}))$ at the correct stress $\left(\mathrm{k}_{\mathrm{AE}}=1\right.$, stress occurs at a constant rate $\dot{\sigma} \neq 0$, the rules of AE testing are observed), the model makes it possible to formulate the energy, structural, and the time strength characteristics corresponding to macro and nano levels, as well as to formulate a 
number of diagnostic AE indicators of the strength state (Table 1), which underlie the assessment of the quality of reinforcing technologies.

Table 1. Concentration-kinetic AE-strength indicators.

\begin{tabular}{llll}
\hline AE indicator & Micromodel & Nanomodel & Macromodel \\
\hline $\mathrm{X}_{\mathrm{AE}}\left(\mathrm{s}^{-1}\right)$ & $d \ln \xi / \mathrm{dt}$ & $\gamma \dot{\sigma} / \mathrm{KT}$ & - \\
$\mathrm{Y}_{\mathrm{AE}}\left(\mathrm{Pa}^{-1}\right)$ & $d \ln \xi / \mathrm{d} \sigma$ & $\gamma / \mathrm{KT}$ & $\mathrm{d} \ln \mathrm{N}_{\mathrm{c}} / \mathrm{d} \sigma^{*}$ \\
$\mathrm{kY}_{\mathrm{AE}}\left(\mathrm{N}^{-1}\right)^{* *}$ & $d \ln \xi / \mathrm{dF}$ & $\mathrm{k} \gamma / \mathrm{KT}$ & $\mathrm{dlnN}_{\mathrm{c}} / \mathrm{dF}$ \\
$\mathrm{W}_{\mathrm{AE}}$ & $d \ln \xi / \mathrm{dK}_{l}^{* * *}$ & & $\operatorname{lnN}_{\mathrm{B}}-\operatorname{lnN}_{\mathrm{w}}{ }^{*}$ \\
\hline
\end{tabular}

$* \mathrm{~N}_{\mathrm{c}}, \mathrm{N}_{\mathrm{B}}, \mathrm{N}_{\mathrm{w}}$ are the slave parameters of the material fatigue curve;

${ }^{* *} \mathrm{k}=\sigma / \mathrm{F}$ is the proportionality coefficient between the stress $\mathrm{F}$ and the nominal stresses $\sigma$,

$* * * K_{l}$ is the stress factor (ratio of the diagnostic stress to the working one).

\section{Experiment and results}

\subsection{Samples, hardening technologies, and equipment}

Practical approbation of the proposed approach was applied on flat steel specimens with a weld. A typical sample is shown in Figure 2a. The specimens were made of steel 3 (EU - Fe37-3FN) with overall dimensions of $150 \times 25 \times 4(\mathrm{LxWxH})$. The location of the weld is in the middle, welding is performed on both sides. Type of welding: semi-automatic. The samples were divided into 5 groups and processed with three types of hardening technologies, such as heat treatment, ultrasonic treatment, and hardening associated with welding. The first group of steel samples consisted of untreated specimens.

\subsubsection{Heat treatment (tempering)}

The second group of steel samples was processed using the hardening technology in the form of heat treatment to relieve residual stresses in welded joints. High tempering was applied to it. The technology shown in Figure 2b was implemented in an SNOL 7.2 / 1100 muffle furnace with heating up to $600^{\circ} \mathrm{C}$ at a heating step of $10^{\circ}$ per minute, with the subsequent exposure at this temperature for 1 hour and cooling along with the furnace. At the same time, the level of hardness also decreases, ductility and toughness increase, residual stresses decrease by $70-80 \%$.

\subsubsection{Ultrasonic processing and chamfer}

The third and fourth groups were processed by ultrasonic impact treatment [5,14], of the weld and the heat-affected zone shown in Figure 2c. The parameters of the sensor, in which the metal was exposed, were at a frequency of $20.1 \mathrm{kHz}$ and a power of $200 \mathrm{~W}$.

The fifth group of samples was affected by welding technology and, in particular, by the presence of a chamfers on both sides of the metal. The technological feature of this type of welding is shown in Figure 2d. The chamfer is designed for good penetration and durable welding of joints, as well as reduced welding time.

\subsubsection{Equipment}

To create a stress-strain state in the samples, the Zwick / Roell Z100 universal testing machine was used, as shown in Figure 3a. The equipment was subjected to tensile tests of steel samples, in which acoustic emission was recorded. Registration of elastic waves was carried out using the 
AE transducers, i.e. piezoceramic tablets made of lead zirconate titanate TsTS-19, placed in a brass case, where they were converted into an electrical signal. The registration was carried out using a two-channel measuring acoustic emission system with subsequent recording of the results on the computer as shown in Figure 3b, c, d. Processing of the obtained results was performed using the approach described in the work.

\subsection{Experiment and processing of results}

Samples of welded joints were stretched to rupture on a testing machine, where acoustic emission was recorded. It was originally planned to compare the data obtained during metal fracture related to the mechanical properties of the material (tensile strength, yield strength, etc.) with acoustic emission data. As a result of the experiment, the rupture of the samples occurred in the base metal, and as a result, the mechanical properties of the metal, rather than the weld, were measured on the machine. Since hardening technologies were applied to weld (except for heat treatment), and acoustic emission was recorded from welds, the authors decided that the comparison would be incorrect.

When processing the results, a section of elastic fracture was used, which corresponds to the working stresses experienced by industrial facilities in reality. An example of elastic fracture and stress of a typical sample are shown in Figure 4. In the section of elastic fracture, from the position of the information and kinetic approach, using computer simulation, a section of homogeneous fracture was selected, shown in Figure 5. At this stage, the values of the concentration and kinetic indicators of the AE strength were calculated according to the formulas from Table 1, which were used to assess the quality of strengthening technologies. The obtained results are presented in Table 2. Both parameters showed the same results when using this approach, while the coefficient of variation between the values of $\mathrm{Y}_{\mathrm{AE}}$ and $\mathrm{X}_{\mathrm{AE}}$ averages $3 \%$. In addition, the obtained results are congruent with the results of cyclic destructive tests described in [15].

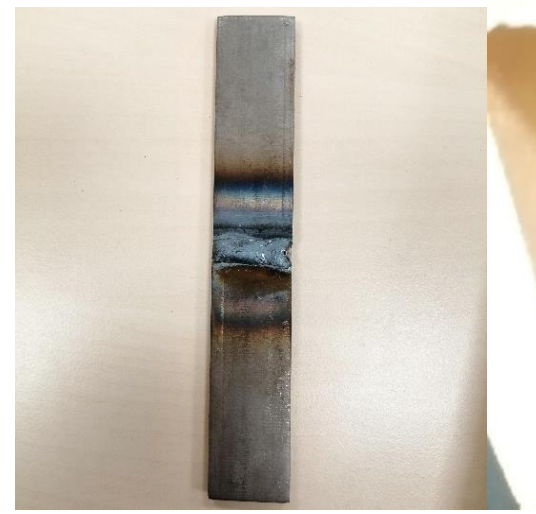

a)

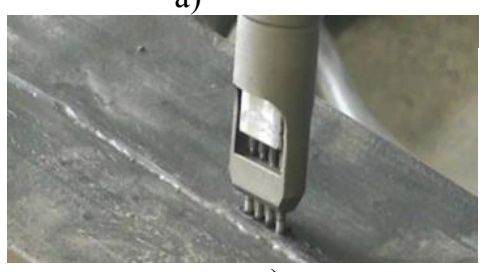

c)

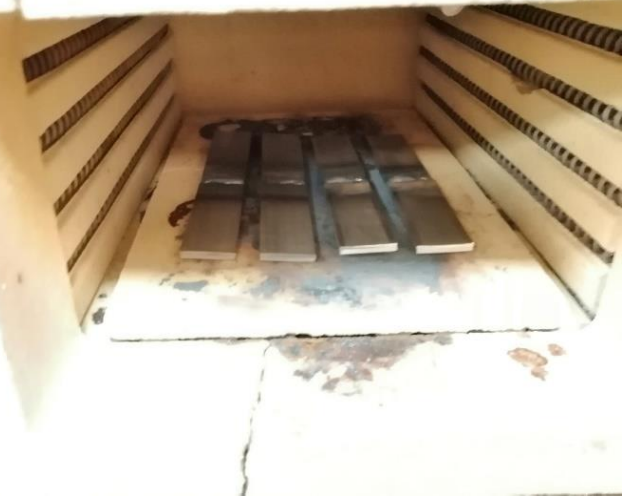

b)

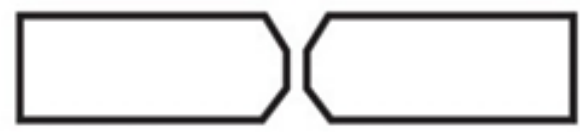

d)

Fig. 2. Sample and hardening technologies: (a) a typical sample, (b) heat treatment of the first group of samples in a furnace, (c) ultrasonic hardening and finishing metal processing, (d) designation of chamfers on the sample (side view). 


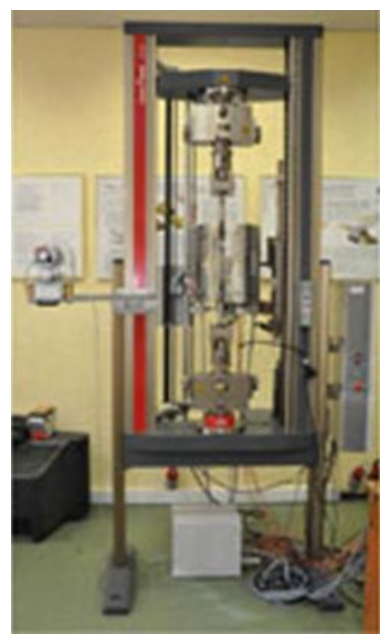

a)

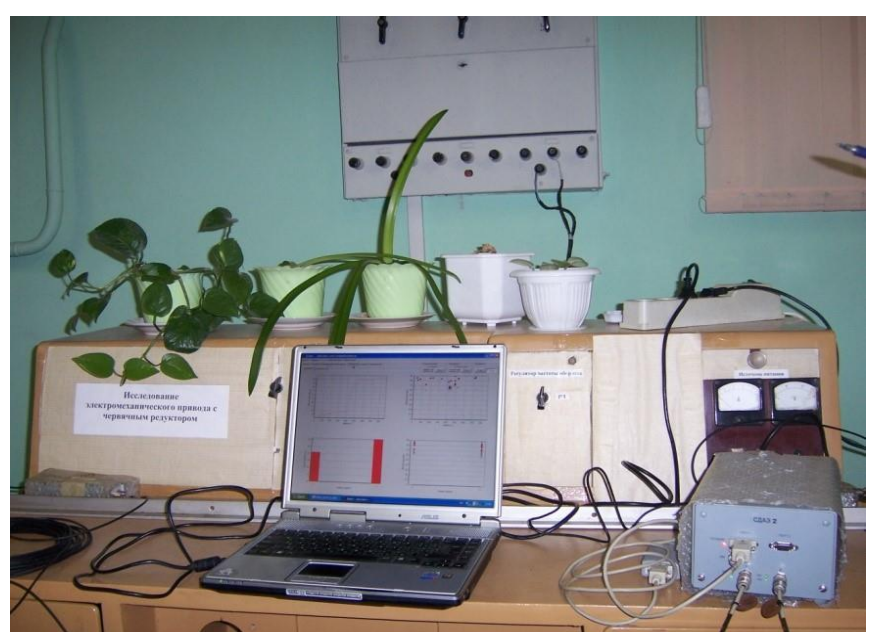

b)

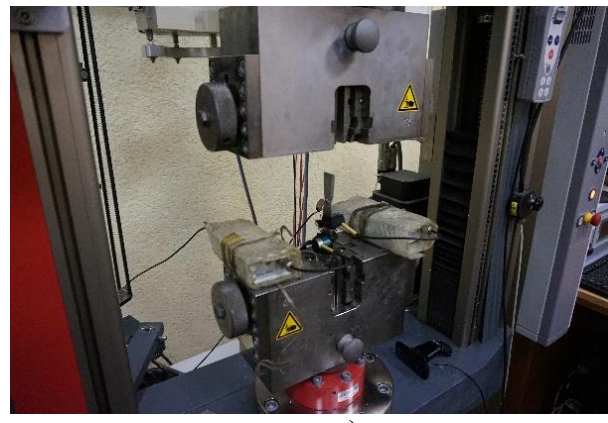

c)

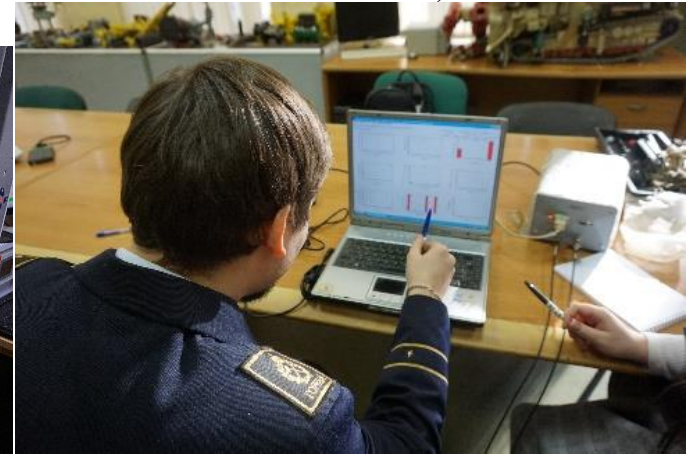

d)

Fig. 3. Equipment used for the experiment: (a) universal testing machine Zwick / Roell Z100; (b) acoustic emission equipment, (c) acoustic emission transducers located on the sample, (c) acoustic emission registration process.

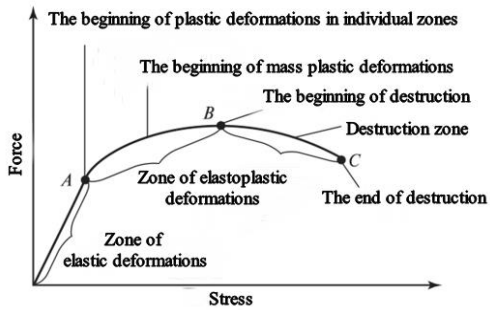

a)

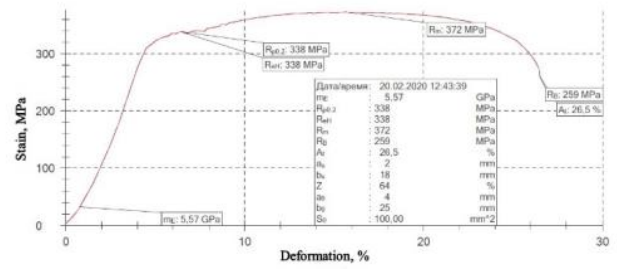

b)

Fig. 4. Graphs of dependences of force on deformation, where: (a) is a diagram of the process of metal deformation by zones, (b) is a typical stress curve of one of the samples. 


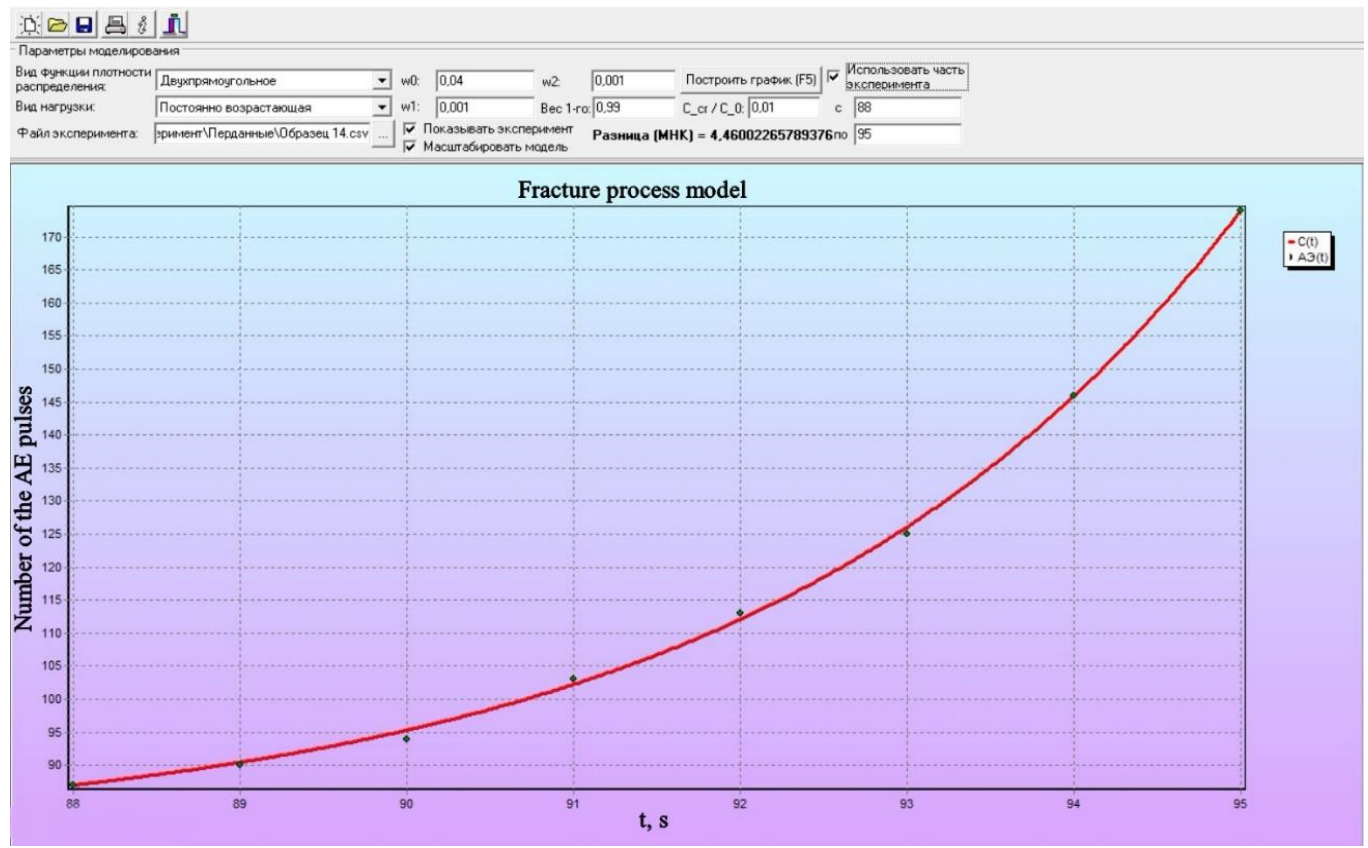

Fig. 5. Screenshot from the programme for modeling the fracture process (red line) and the number of AE pulses (points).

Table 2. Results obtained.

\begin{tabular}{cccccc}
\hline $\begin{array}{c}\text { Type of HT / parameter (di- } \\
\text { mension) }\end{array}$ & Untreated & $\begin{array}{c}\text { Heat } \\
\text { treatme } \\
\text { nt }\end{array}$ & $\begin{array}{c}\text { With } \\
\text { chamfer }\end{array}$ & $\begin{array}{c}\text { UST } \\
\text { of } \\
\text { weld }\end{array}$ & $\begin{array}{c}\text { UST of heat- } \\
\text { affected area }\end{array}$ \\
\hline $\mathrm{X}_{\mathrm{AE}}\left(\mathrm{s}^{-1}\right)$ & 0,066 & 0,025 & 0,036 & 0,044 & 0,072 \\
$\mathrm{Y}_{\mathrm{AE}}\left(\mathrm{MPa}^{-1}\right)$ & 0,0042 & 0,0012 & 0,0028 & 0,003 & 0,0058 \\
& 6 & 3 & 1 & 3 & 1 \\
$\begin{array}{c}\text { The difference between the } \\
\text { coefficients of variation of } \\
\mathrm{Y}_{\mathrm{AE}} \text { and } \mathrm{X}_{\mathrm{AE}}(\%)\end{array}$ & & & & & \\
\hline
\end{tabular}

\section{Conclusion}

As a result of the work done, the analysis of the quality control methods of hardening technologies was carried out using the example of a weld and an information- kinetic approach to assessing the quality of hardening technologies was considered. Based on the results of the work, the following conclusions were made:

1. The proposed method, based on a multi-level model, allows to quickly assess the quality of hardening technologies that affect the entire volume of the product.

2. The attenuation of the AE activity or its absence before the stage of homogeneous destruction from the position of the model is interpreted as the removal of internal stresses, but is not associated with the strengthening of structural elements.

3. Hardening occurs where the values of concentration and kinetic strength indicators $\left(\mathrm{X}_{\mathrm{AE}}\right.$, $\left.\mathrm{Y}_{\mathrm{AE}}\right)$ are reduced. The lower the parameter values, the better the hardening. 
4. The increased AE activity of hardened heat-treated samples indicates the low information content of the number of pulses, MARS and the total AE amplitude, traditionally used to assess the degree of danger of the state.

5. The excess of the values of concentration and kinetic strength indicators for HT UST of the heat-affected zone can be explained by its softening effect. The near-heat zone itself is hardened and has low values of concentration and kinetic AE indicators.

\section{References}

1. V.I. Bolobov, E.A. Krivokrisenko, G.G. Popov, V.E. Nikulin, Determination of residual stresses in metal structures by using magnetic anisotropy, Modern educational technologies in training specialists for the mineral and raw materials complex. Collection of scientific papers of the 3rd All-Russian scientific conference, 1004-1009 (2020)

2. V.V. Maksarov, E.V. Koshaleva, A.Yu. Vazhenin, Increasing the quality of production of parts from titanium alloys by the method of local plastic deformation, Bulletin of P.A. Solovyov Rybinsk State Aviation Technical University, 1(40), 276-281 (2017).

3. V. V. Gabov, N. V. Babyr, D. A. Zadkov Mathematical modelling of operation of the hydraulic support system of the powered support sections with impulse-free continuous regulation of its resistance to the roof rock lowering, IOP Conference Series: Materials Science and Engineering, 1064, (2021).

4. M.A. Filippov, The methodology of the choice of metal alloys and hardening technologies in mechanical engineering. (Yekaterinburg: Publishing House of the Ural University, 2013).

5. A.G. Palaev, A.I. Potapov, Technology, equipment for ultrasonic hardening and finishing of metals, and quality control, Metalworking, 6, 38 - 41 (2018).

6. G.A. Iovlev Method of graphical representation of yield and failure criteria in the HaighWestergaard principal stress space, Modern educational technologies in training specialists for the mineral and raw materials complex. Collection of scientific papers of the 3rd AllRussian scientific conference, 1539-1548 (2020).

7. S.I. Buylo Physicomechanical, statistical and chemical aspects of the acoustic emission diagnostics. (Rostov-on-Don: Publishing House of SFU, 2017).

8. P. Feng, P. Borghesani, W. A Smith, Z. Peng, Model-based surface roughness estimation using acoustic emission signals, Tribology International, 144, 2020.

9. V.C. Kachanov, I.V. Sokolov, V.M. Matyunin, V.A. Barat, V.V. Bardakov, A. A. Marchenkov, Evaluation of crack resistance of strengthening titanium nitride coatings using the parameters of kinetic indentation and acoustic emission, Measurement Techniques, 7, 41-44 (2017).

10. D.L. Merson, M.A. Vagapov The special role of the surface in deformation processes and acoustic radiation of metals and alloys, Tomsk State University Journal, 15(3), 796-799 (2010).

11. I.V. Miroshin, Technique of acoustic emission studies of the inherited quality parameters of the surface layer under the conditions of cutting and surface plastic deformation, Bulletin of the Kuzbass State Technical University, 3, 46-52 (2010).

12. V.V. Nosov, Control of inhomogeneous materials strength by the method of acoustic emission, Journal of Mining Institute, 226, 469-479 (2017).

13. J. Hu, T. Shimizu, T. Yoshino, T. Shiratori, M. Yang, Evolution of acoustic softening effect on ultrasonic-assisted micro/meso-compression behavior and microstructure, Ultrasonic, 107, 1-11 (2020). 
14. A.I. Potapov, V.E Makhov, Methods for non-destructive testing and diagnostics of durability of articles made of polymer composite materials, Russian Journal of Nondestructive Testing, 54(3), 151-163 (2018).

15. K.D. Verzhbidsky, Provision of the resource of a vertical steel tank by additional processing of welded joints of the lower belt. (St. Petersburg: St. Petersburg Mining University, 2018). 